\title{
Direct Analysis of Conjugate Metabolites of Methamphetamine, 3,4-Methylenedioxymethamphetamine, and Their Designer Drugs in Biological Fluids
}

\author{
Noriaki Shima, ${ }^{*}$ Munehiro Katagi, and Hitoshi Tsuchihashi \\ Forensic Science Laboratory, Osaka Prefectural Police Headquarters, 1-3-18 Hommachi, Chuo-ward, Osaka 541-0053, Japan
}

(Received March 20, 2009)

\begin{abstract}
This review summarizes technical improvements in the forensic analysis for conjugate metabolites of methamphetamine (MA), 3,4-methylenedioxymethamphetamine (MDMA) and their designer drugs. The improvements include the establishments of liquid chromatography-mass spectrometry-mass spectrometry (LC-MS-MS) in combination with the pretreatment methods. The direct determination techniques for the conjugates proved to be effective in rapidly and accurately examining many biological specimens without annoying hydrolysis. The metabolism of MA and MDMA, with attention to the phase II metabolism of the major hydroxy metabolites, is also discussed based on concentration data in biological specimens obtained from drug abusers.
\end{abstract}

Key words — methamphetamine, 3,4-methylenedioxymethamphetamine, conjugate metabolite, liquid chromatography-mass spectrometry, direct analysis

\section{INTRODUCTION}

Drug abuse, which affects human nature and causes numerous crimes, is a serious problem throughout the world. Methamphetamine (MA) and 3,4-methylenedioxymethamphetamine (MDMA) are among the most prevalent of illicit drugs used in Asian countries. ${ }^{1-5)}$ Recently, the abuse of their designer drugs including $p$-methoxyamphetamine (PMA) analogues and 2-methylamino-1-(3,4methylenedioxyphenyl)-propan-1-one (methylone) analogues, as shown in Fig. 1, have also been on the rise in Japan as well as in Europe and the U.S.A. ${ }^{6-12)}$ More recently, the Japanese authorities, therefore, have begun to prevent not only the recreational uses of the designer drugs under Narcotics and Psychotropics Control Law, but also the importation and distribution under Pharmaceutical Affairs Law.

Phenethylamine analogues such as MA and MDMA are well-known as a potent releaser and/or reuptake inhibitor of presynaptic serotonin, dopamine and norephedrine, and these actions

\footnotetext{
*To whom correspondence should be addressed: Forensic Science Laboratory, Osaka Prefectural Police Headquarters, 1-3-18 Hommachi, Chuo-ward, Osaka 541-0053, Japan. Tel.: +81-6-6268-1234; Fax: +81-6-6271-8066; E-mail: nori6499@amber.plala.or.jp
}

contribute stimulation of the central nervous system, desires to socialize and/or hallucinatory effects. ${ }^{13-17)}$ These pharmacological effects lead to the strong drug addictions, which are causing serious problems such as death, murder, robbery and the spread of infectious diseases.

To combat the use of such drugs, the analysis for each parent drug and its metabolites in biological specimens including urine, blood and hair are becoming indispensable tools of drug enforcement procedures worldwide. ${ }^{18-25)}$ Especially, the determinations of specific metabolites in addition to unchanged drugs provide absolute proof of drug intakes. MA, MDMA and their designer drugs are metabolized mainly to $p$-hydroxylated or demethylenated (followed by methylation) form as shown in Fig. 1. 14,26-30) The main metabolites are generally effective in detecting as indicators of drug abuses because they retain structural characteristics of the parent compound and are detectable for longer period. In human, the majority of main metabolites are immediately conjugated to the glucuronides and/or sulfates, and excreted in urine. Therefore, to more effectively detect the metabolites, urine specimens have generally been hydrolyzed using acids and $\beta$ glucuronidase prior to the instrumental analysis, 
(A)
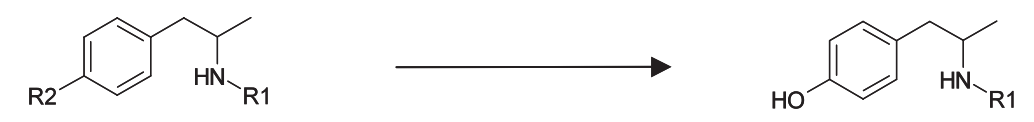

Parent drugs

Main metabolites

\begin{tabular}{lccc}
\hline \hline Drugs & $\mathrm{R} 1$ & $\mathrm{R} 2$ & Regulation in Japan \\
\hline AP & $\mathrm{H}$ & $\mathrm{H}$ & Stimulants \\
MA & $\mathrm{CH}_{3}$ & $\mathrm{H}$ & Stimulants \\
PMA & $\mathrm{H}$ & $\mathrm{OCH}_{3}$ & Narcotics \\
PMMA & $\mathrm{CH}_{3}$ & $\mathrm{OCH}_{3}$ & Scheduled drugs under the pharmaceutical affairs law \\
PMEA & $\mathrm{C}_{2} \mathrm{H}_{5}$ & $\mathrm{OCH}_{3}$ & Nonregulated \\
\hline
\end{tabular}

(B)

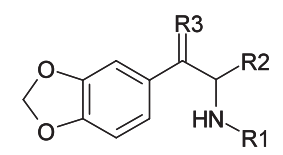

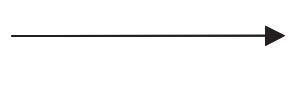

Parent drugs

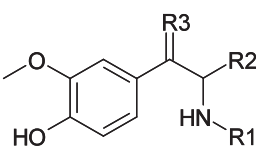

Main metabolites

\begin{tabular}{lcccc}
\hline \hline Drugs & $\mathrm{R} 1$ & $\mathrm{R} 2$ & $\mathrm{R} 3$ & Regulation in Japan \\
\hline MDA & $\mathrm{H}$ & $\mathrm{CH}_{3}$ & $\mathrm{H}_{2}$ & Narcotics \\
MDMA & $\mathrm{CH}_{3}$ & $\mathrm{CH}_{3}$ & $\mathrm{H}_{2}$ & Narcotics \\
MDEA & $\mathrm{C}_{2} \mathrm{H}_{5}$ & $\mathrm{CH}_{3}$ & $\mathrm{H}_{2}$ & Narcotics \\
MBDB & $\mathrm{CH}_{3}$ & $\mathrm{C}_{2} \mathrm{H}_{5}$ & $\mathrm{H}_{2}$ & Narcotics \\
Methylone & $\mathrm{CH}_{3}$ & $\mathrm{CH}_{3}$ & $\mathrm{O}$ & Narcotics \\
bk-MDEA & $\mathrm{C}_{2} \mathrm{H}_{5}$ & $\mathrm{CH}_{3}$ & $\mathrm{O}$ & Scheduled drugs under the pharmaceutical affairs law \\
bk-MBDB & $\mathrm{CH}_{3}$ & $\mathrm{C}_{2} \mathrm{H}_{5}$ & $\mathrm{O}$ & Scheduled drugs under the pharmaceutical affairs law \\
\hline
\end{tabular}

Fig. 1. Compound Structures and Their Major Metabolic Pathways of (A) AP and (B) MDA Analogues

AP, Amphetamine; PMMA, p-Methoxymethamphetamine; PMEA, p-Methoxyethylamphetamine; MDA, 3,4-Methylenedioxyamphetamine; MBDB, N-Methyl-1-(1,3-benzodioxol-5-yl)-2-butanamine; Methylone, 2-Methylamino-1-(3,4methylenedioxyphenyl)propan-1-one; bk-MDEA, 2-Ethylamino-1-(3,4methylenedioxyphenyl)propan-1-one; bk-MBDB, 2-Methylamino-1-(3,4methylenedioxyphenyl)butan-1-one.

gas chromatography-mass spectrometry (GC-MS) and/or liquid chromatography-mass spectrometry (LC-MS). ${ }^{31,32)}$ The hydrolysis, however, has disadvantages such as requiring tedious handling and time-consuming incubation as well as obscuring the conjugation patterns of metabolites. The direct determination of the conjugate metabolites, if feasible, can resolve these problems without incident. Its application to forensic biological specimens has been, indeed, published: Maurer et al. reported the detection of conjugate metabolites of 3,4-methylenedioxyethylamphetamine (MDEA) by using GC-MS and LC-MS techniques. ${ }^{33)}$ It was, however, uncertain that these procedures including the extractions provide enough sensitivity and quantitative capability to be applied to the forensic and toxicological analysis. More recently, the authors have optimized the direct determination methods for the conjugated metabolites of MA and MDMA by using LC-MS and LC-MS-MS, ${ }^{18,34,35)}$ and have evaluated those accurate concentrations in urine and blood obtained from MA and MDMA users. ${ }^{18,26,27)}$ This review summarizes technical improvements for conjugate metabolites and the studies on phase II metabolism of MA and MDMA based on the concentration data.

\section{DIRECT DETERMINATION OF CONJUGATE METABOLITES}

\section{Sample Preparation for Urine and Blood Speci- mens}

The sample pretreatment is one of the most essential processes to accurately and sensitively determine conjugate metabolites. The solid-phase extractions (SPE) or liquid-liquid extractions have been generally adopted as the pretreatments for phenethylamine analogues themselves. There have been, however, no SPE cartridges and extracting solvents that provide high recoveries for their conjugate metabolites because of their high polarities.

Maurer et al. reported that 4-hydroxy-3methoxyethylamphetamine (HMEA, main metabolite of MDEA shown in Fig. 1) glucuronide and 3,4-dihydroxyethylamphetamine (DHEA, metabolites of MDEA) sulfate were isolated from human urine by SPE on a Bond Elut C18 cartridge (Varian, Palo Alto, CA, U.S.A) with methanol elution, and were respectively detected by GC-MS and LC-MS. ${ }^{33)}$ Its recoveries were, however, unmentioned because of the unavailability of the authentic standard for the conjugates.

More recently, the authors have synthe- 
sized both the glucuronides and the sulfates of $p$-hydroxymethamphetamine ( $p$-OHMA, main metabolite of MA) and 4-hydroxy-3-methoxymethamphetamine (HMMA, main metabolite of MDMA), and have examined several SPE and protein precipitation method with methanol by analyzing spiked urine and blood samples with the authentic conjugates. The protein precipitation method for LC-MS and LC-MS-MS provided high recoveries of not less than $78 \%$ for the conjugates as well as other metabolites of MA and MDMA. ${ }^{18,34,35)}$ The technique is extremely simple and rapid, while it allowed us to analyze without any significant disturbance by impurities in urine and blood. Contrastively, none of common-type SPE cartridges provided quantitative retention of the conjugates. ${ }^{34)}$

Kamata et al. and Zaitsu et al. have also successfully applied the precipitation method to pretreatment procedures for LC-MS and LC-MSMS analyses of the conjugates of 4-hydroxy-3methoxymethcathinone (HMMC, main metabolite of methylone) and $p$-hydroxyethylamphetamine [ $p$-OHEA, main metabolite of $p$-methoxyethylamphetamine (PMEA)]. ${ }^{20,29)}$

\section{LC-MS and LC-MS-MS}

The free forms of main hydroxylated metabolites of phenethylamine analogues are analyzed by not only LC-MS but also GC-MS with trifluoroacetyl derivatization procedures. ${ }^{19,21)}$ Until recently, there had been, however, no reports that described direct detections of their conjugates by either LC-MS or GC-MS.

Recently, Maurer et al. reported the detection of HMEA-glucuronide as the trimethylsilyl derivative by GC-MS. ${ }^{33)}$ It is, however, uncertain that this technique provides enough sensitivity of detection and quantitative capability because the derivative reaction is inefficient and the resulting formed derivative is significantly labile. Maurer et al. have also detected DHEA-sulfate in human urine by LC-MS in the negative mode. ${ }^{33)}$ In theory, an LC-MS technique has advantages over GC-MS in the sensitive detections of polar compounds including conjugate metabolites.

The glucuronide of phenethylamine analogues contains both an amino and a carboxyl group, and the sulfate has an amino and a sulfate group. The amino group is easily protonated, while the carboxyl and sulfate groups are rapidly deprotonated. The selection of a suitable ionization mode for
LC-MS techniques is, therefore, indispensable for the sensitive detection of the conjugates.

The authors developed electrospray ionization (ESI)-MS and ESI-MS-MS conditions for direct determination by utilizing the authentic conjugates, $p$-OHMA-glucuronide ( $p$-OHMAGlu), $p$-OHMA-sulfate ( $p$-OHMA-Sul), HMMAglucuronide (HMMA-Glu) and HMMA-sulfate (HMMA-Sul) described in previous section. ${ }^{18,34,35)}$ The predominant $[\mathrm{M}+\mathrm{H}]^{+}$and $[\mathrm{M}-\mathrm{H}]^{-}$ions were respectively observed in the positive and negative modes, and the 5 to 30 times higher sensitivities for the sulfate and glucuronide was obtained in the positive mode than in the negative mode under the conditions of acidified mobile phase ( $\mathrm{pH} 3.5$ ). Figure 2 shows the product ion spectra of $p$-OHMA-Glu, p-OHMA-Sul, HMMA-Glu and HMMA-Sul obtained from an MA and MDMA user's blood specimen. On selecting each protonated molecule as a precursor ion, the product ions at $m / z 166$ and 196 were respectively observed by the loss of 176 of the glucuronyl group and 80 of the sulfonate group. Additionally, the characteristic substructural ions of $p$-OHMA and HMMA at $m / z 135$ and 165 were respectively observed. ${ }^{18)}$

The authors have also developed the LC chromatography conditions employing a C18 semimicro column with linear gradient eluent programs, which provided successful elutions of MA and its four metabolites, and MDMA and its five metabolites approximately within $20 \mathrm{~min}$. To demonstrate the forensic utility of the LC-MS and LC-MS-MS methods, the validation tests were accomplished by analyzing spiked urine and blood samples with the authentic conjugates. ${ }^{18,34,35)}$ The limits of detection (LODs) of these analytes were approximately $5 \mathrm{ng} / \mathrm{ml}$ and $2 \mathrm{ng} / \mathrm{ml}$ in the LC-MS (the selectedion monitoring mode) and LC-MS-MS (the selected reaction monitoring mode) techniques, respectively. The obtained validation data guaranteed high sensitivity and quantitative efficiencies of the methods including the pretreatment.

\section{APPLICATION FOR FORENSIC TOXICOLOGICAL SPECIMENS}

\section{MA and MDMA}

In early studies on the metabolism of MA and MDMA, the indirect determinations of conjugates of $p$-OHMA and HMMA have been reported by comparing the results before and after hydrolysis 
(A)

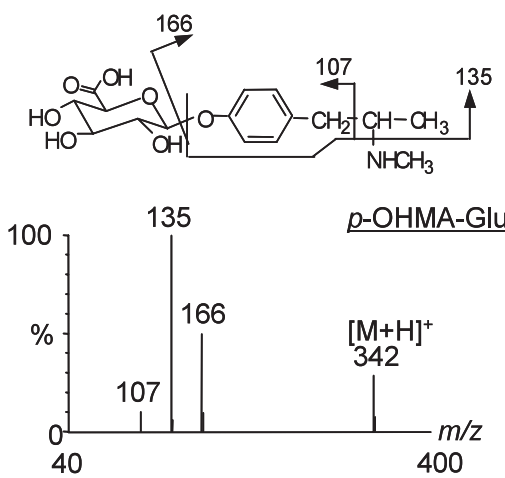

(C)

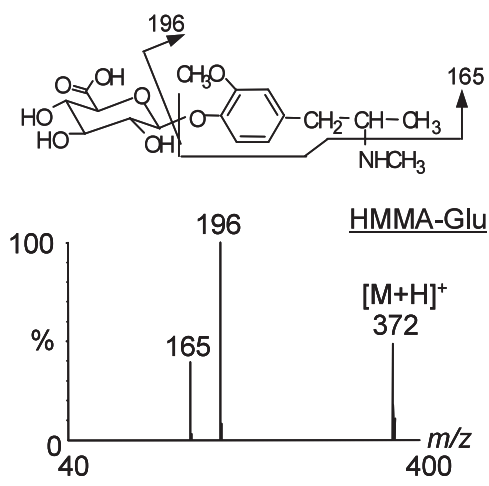

(B)

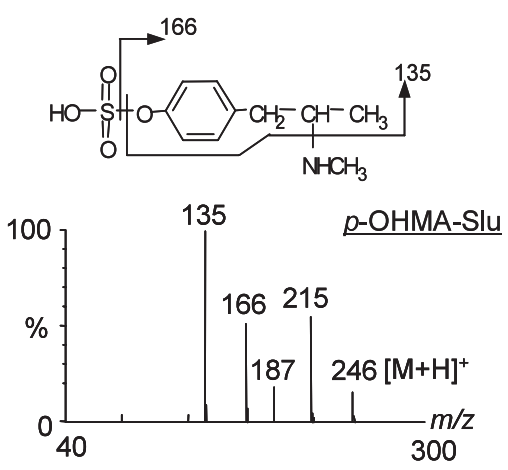

(D)
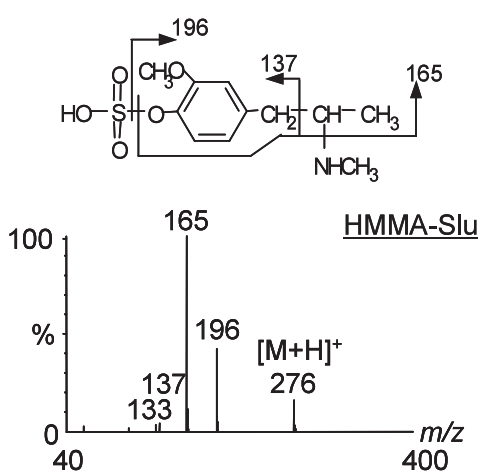

Fig. 2. Product Ion Mass Spectra of Conjugate Metabolites of MA and MDMA, (A) p-OHMA-Glu, (B) p-OHMA-Sul, (C) HMMASul and (D) HMMA-Glu

ESI-MS-MS was carried out in the positive ion modes, and each protonated molecule was selected as a precursor ion.

Table 1. Concentrations of the Main Metabolites and Its Conjugates of MA and MDMA in Urine and Blood

\begin{tabular}{|c|c|c|c|c|c|c|}
\hline & \multicolumn{6}{|c|}{ MA users } \\
\hline & \multicolumn{3}{|c|}{ Urine specimens $(\mu \mathrm{g} / \mathrm{ml}, n=50)$} & \multicolumn{3}{|c|}{ Blood specimens (ng/ml, $n=11)$} \\
\hline & $p$-OHMA (free) & $p$-OHMA-Sul & $p$-OHMA-Glu & $p$-OHMA (free) & $p$-OHMA-Sul & $p$-OHMA-Glu \\
\hline Range & $<0.005-1.3$ & $0.02-21.7$ & $<0.02-2.4$ & $<1.0-131$ & $3.8-495$ & $<2.0-46.7$ \\
\hline \multirow[t]{4}{*}{ Mean \pm S.D. } & $0.4 \pm 0.4$ & $3.2 \pm 3.9$ & $0.4 \pm 0.5$ & $23.5 \pm 39.6$ & $97.9 \pm 136$ & $6.3 \pm 13.7$ \\
\hline & \multicolumn{6}{|c|}{ MDMA users } \\
\hline & \multicolumn{3}{|c|}{ Urine specimens $(\mu \mathrm{g} / \mathrm{ml}, n=25)$} & \multicolumn{3}{|c|}{ Blood specimens (ng/ml, $n=3)$} \\
\hline & HMMA (free) & HMMA-Sul & HMMA-Glu & HMMA (free) & HMMA-Sul & HMMA-Glu \\
\hline Range & $0.04-2.3$ & $1.8-55.6$ & $0.5-32.3$ & $<1.0-84.2$ & $190-610$ & $78.1-508$ \\
\hline Mean \pm S.D. & $0.5 \pm 0.5$ & $15.5 \pm 11.8$ & $8.2 \pm 7.0$ & $45.5 \pm 54.4$ & $464 \pm 238$ & $242 \pm 232$ \\
\hline
\end{tabular}

although the conjugation pattern remained to be ambiguous. $^{32,36,37)}$ More recently, the authors have analyzed 75 urine specimens (obtained from 50 MA and $25 \mathrm{MDMA}$ users) and 14 blood specimens (obtained from $11 \mathrm{MA}$ and 3 MDMA users) submitted for forensic analysis in order to directly and accurately evaluate the concentration of $p$-OHMAGlu, p-OHMA-Sul, HMMA-Glu and HMMA-Sul in biological fluids. ${ }^{18,26,27)}$ Table 1 summarizes the results including ranges and averages of those concentrations, and Fig. 3 shows typical extracted mass chromatograms obtained from an MA and
MDMA user's urine specimen along with rat urine specimens.

The concentration of $p$-OHMA-Sul and HMMA-Sul was respectively higher than that of each glucuronide in all of the specimens $(n=89)$ obtained from MA and MDMA users, and the molar ratios of sulfate to glucuronide (hereafter, S/G ratio) ranged from 2.2 to 37.1 (mean \pm S.D.: $13.8 \pm 8.1$, $n=41$ ) for MA users' urine, and from 1.6 to 9.9 (mean \pm S.D.: $3.13 \pm 1.81, n=25)$ for MDMA users' urine, and from 11.8 to 46.6 (mean \pm S.D.: $28.2 \pm 16.3, n=6$ ) for MA users' blood, and from 

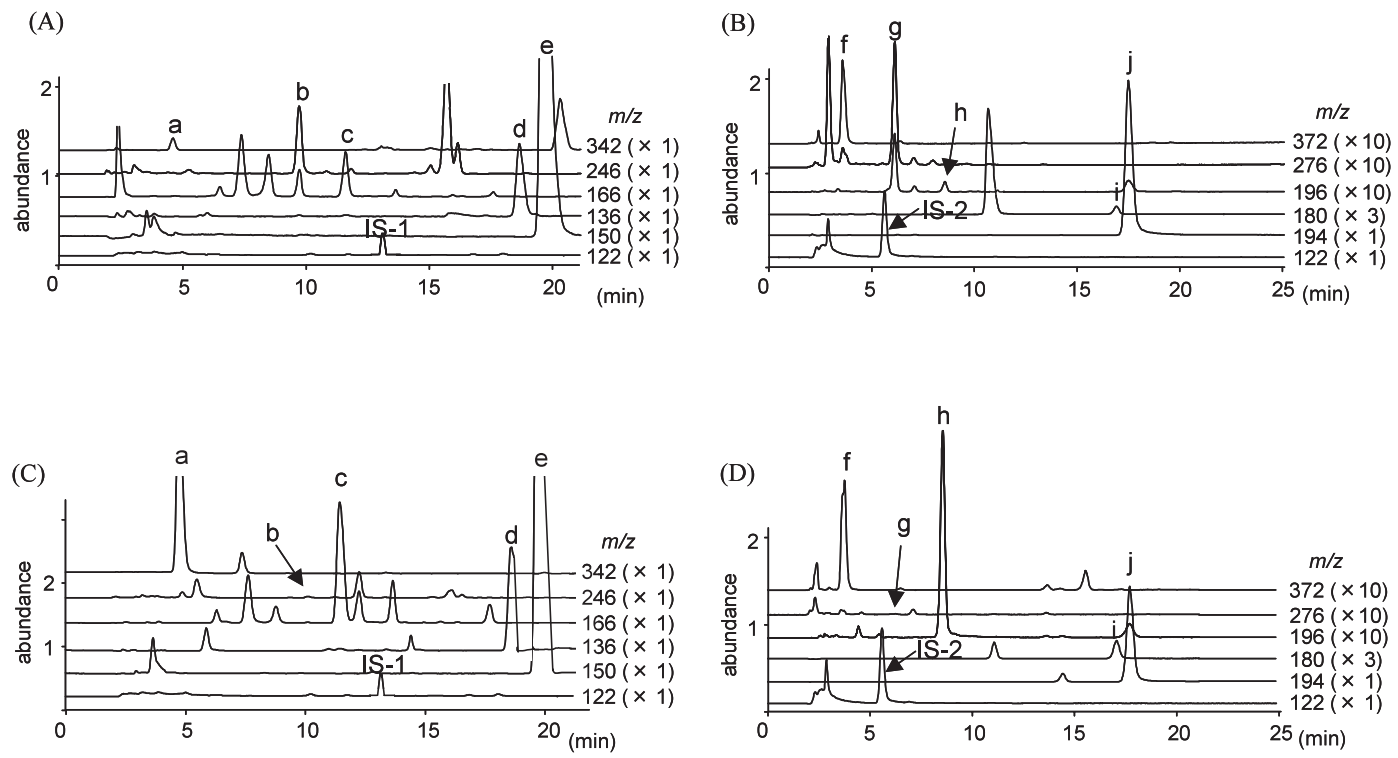

Fig. 3. Extracted Mass Chromatograms Obtained from Human Urine (A, MA intake; B, MDMA intake) and Rat Urine (C, MA administrated; D, MDMA administrated)

LC was performed at each condition for MA and MDMA, and ESI-MS was carried out in the selected-ion monitoring mode. The numbers in parentheses represent magnifications. Peaks: (a), p-OHMA-Glu; (b), p-OHMA-Sul; (c), p-OHMA; (d), AP; (e), MA; (f), HMMA-Glu; (g), HMMA-Sul; (h), HMMA; (i), MDA; (j), MDMA; (IS-1), $\beta$-phenethylamine (internal standard); (IS-2), N-methylbenzylamine (internal standard).

1.6 to 5.7 (mean \pm S.D.: $3.52 \pm 2.03, n=3$ ) for MDMA users' blood. ${ }^{18,26,27)}$

Also, the molar conjugation percentage ([sulfate + glucuronide]/[free form + sulfate + glucuronide] $\times 100 \%$ ) ranged from 59.3 to $100 \%$ (mean \pm S.D.: $85.3 \pm 8.3 \%, n=50$ ) for MA users' urine, from 72.7 to $99.3 \%$ (mean \pm S.D.: $95.6 \pm 6.0 \%$, $n=25$ ) for MDMA users' urine, from 64.9 to $100 \%$ (mean \pm S.D.: $85.0 \pm 12.0 \%, n=10$ ) for MA users' blood, from 89.3 to $100 \%$ (mean \pm S.D.: $95.2 \pm 5.4 \%$, $n=3$ ) for MDMA users' blood. ${ }^{18,26,27)}$ These data indicated that $p$-OHMA and HMMA derived from MA and MDMA were predominantly conjugated to form its sulfate in humans, respectively. Thus, each sulfate is effective in directly detecting as an indicator of the abuses, which unequivocally proves the intakes of MA or MDMA, because it is expected to be contained in urine and blood at the higher concentration than other metabolites in many cases. Additionally, when compared the concentrations in urine and blood, the trends of conjugation pattern in blood are very similar to those in urine. However, the blood specimens submitted for proof of drug abuse or poisoning had not been usually hydrolyzed prior to the instrumental analysis. It is probably because the significance of such conjugated metabolites in blood had not been recognized until recently. These findings suggest that hydrolysis of blood specimens is essential to measure total levels of $p$-OHMA or HMMA as toxicological indicator.

On the other hand, in the rats, $\mathrm{S} / \mathrm{G}$ ratios were much lower than in the humans because almost all of the conjugated $p$-OHMA and HMMA (>99\%) was excreted as the glucuronide in urine, ${ }^{26,27)}$ which indicates that a considerable interspecies variation exists in the conjugation of $p$-OHMA and HMMA between humans and rats as shown in Fig. 3.

\section{Designer Drugs}

Several forensic studies on conjugate metabolites of designer drugs have been performed by modifying the LC-MS and LC-MS-MS procedures established by authors.

Zaitsu et al. reported that the glucuronide and sulfate of $p$-OHEA (main metabolites of PMEA) were directly detected in human urine by LC-MSMS. Although these urinary concentrations are unclear because of the unavailability of the authentic standard for the conjugates, the increased amount of $p$-OHEA after hydrolysis indicated that main metabolite were slightly excreted as the conjugates. ${ }^{29)}$ Zaitsu et al. and Kamata et al. have also reported that glucuronide and/or sulfate of main metabolites of bk-MDEA, bk-MBDB, 4chloro-2,5-dimethoxyamphetamine (DOC) and 2,5dimethoxy-4-iodoamphetamine (DOI) were respec- 
tively detected in human urine by LC-MS and LC-MS-MS, which estimated that the main metabolites were eliminated partially as conjugates. ${ }^{30,38)}$ On the other hand, Kamata et al. demonstrated that, in the Wistar rats, the majority of HMMC (main metabolite of methylone) was eliminated predominantly as its glucuronides based on the ion intensity of the glucuronide, which was directly detected by an LC-MS method. ${ }^{28)}$ Additionally, these reports suggested that $\beta$-keto derivatives such as methylone, bk-MDEA and bk-MBDB are themselves conjugated to unidentified compounds based on the observations of their fractional increase after hydrolysis. ${ }^{28,30)}$

These findings suggest that the interspecies variation and the subtle differences in compound structures result in the slight/large differences in phase II metabolism such as the $S / G$ ratios and conjugation percentages.

\section{CONCLUSION}

The main metabolites of phenethylamine analogues are immediately conjugated to the sulfates and/or glucuronides. To simply identify such conjugate metabolites, the authors have developed the direct determination methods for conjugate metabolites of MA and MDMA. In the forensic field, these techniques proved to be effective in rapidly and accurately examining many biological specimens without annoying hydrolysis, although the availability of authentic standards for conjugates will be needed in order to popularize these techniques as general methods. Additionally, the concentration data in urine and blood obtained from MA and MDMA users demonstrate that the sulfation is quantitatively more significant than the glucuronidation in the phase II metabolism for MA and MDMA in humans. These findings will also contribute to the ulterior studies in various fields such as toxicology, metabolomics and pharmacokinetics.

\section{REFERENCES}

1) National Police Agency of Japan Ed. (2006) White Paper on Police, National Police Agency, Tokyo, Japan.

2) Kulsudjarit, K. (2004) Drug problem in southeast and southwest Asia. Ann. N.Y. Acad. Sci., 1025,
446-457.

3) McKetin, R., Kozel, N., Douglas, J., Ali, R., Vicknasingam, B., Lund, J. and Li, J. H. (2008) The rise of methamphetamine in Southeast and East Asia. Drug Alcohol Rev., 27, 220-228.

4) Chung, H., Park, M., Hahn, E., Choi, H., Choi, H. and Lim, M. (2004) Recent trends of drug abuse and drug-associated deaths in Korea. Ann. N. Y. Acad. Sci., 1025, 458-464.

5) Fang, Y. X., Wang, Y. B., Shi, J., Liu, Z. M. and Lu, L. (2006) Recent trends in drug abuse in China. Acta Pharmacologica Sinica, 27, 140-144.

6) Katagi, M. and Tsuchihashi, H. (2002) Update on clandestine amphetamines and their analogues recently seen in Japan. J. Health Sci., 48, 14-21.

7) Uchiyama, N., Kikura-Hanajiri, R., Kawahara, N. and Goda, Y. (2008) Analysis of designer drugs detected in the products purchased in fiscal year 2006. Yakugaku Zasshi, 128, 1499-505 (in Japanese).

8) Zaitsu, K., Katagi, M., Kamata, H. T., Miki, A. and Tsuchihashi, H. (2008) Discrimination and identification of regioisomeric $\beta$-keto analogues of 3,4-methylenedioxyamphetmaines by gas chromatography-mass spectrometry. Forensic Toxicology, 26, 45-51.

9) Becker, J., Neis, P., Rohrich, J. and Zorntlein, S. (2003) A fatal paramethoxymethamphetamine intoxication. Leg. Med. (Tokyo) Suppl., 1, 138-141.

10) Johansen, S. S., Hansen, A. C., Muller, I. B., Lundemose, J. B. and Franzmann, M. B. (2003) Three fatal cases of PMA and PMMA poisoning in Denmark. J. Anal. Toxicol., 27, 253-256.

11) Casale, J. F., Hays, P. A., Spratley, T. K. and Smith, P. R. (2006) The characterization of 4-methoxy-N-ethylamphetamine hydrochloride. Microgram Journals, 4, 42-46.

12) Bossong, M. G., Van Dijk, J. P. and Niesink, R. J. (2005) Methylone and mCPP, two new drugs of abuse? Addiction Biology, 10, 321-323.

13) Maurer, H. H., Kraemer, T., Springer, D. and Staack, R. F. (2004) Chemistry, pharmacology, toxicology, and hepatic metabolism of designer drugs of the amphetamines (ecstasy), piperazine, and pyrrolidinophenone types: a synopsis. Ther. Drug Monit., 26, 127-131.

14) Green, A. R., Mechan, A. O., Elliott, J. M., O'shea, E. and Colado, M. S. (2003) The pharmacology and clinical pharmacology of 3,4-(methylenedioxy)methamphetamine (MDMA, "Ecstasy"). Pharmacol. Rev., 55, 463-508.

15) Cozzi, N. V., Sievert, M. K., Shulgin, A. T., Jacob, III P. and Ruoho, A. E. (1999) Inhibition of plasma membrane monoamine transporters by $\beta$ - 
ketoamphetamines. Eur. J. Pharmacol., 381, 63-69.

16) Dal Cason, T. A., Young, R. and Glennon, R. A. (1997) Cathinone: An investigation of several $\mathrm{N}$-alkyl and methylenedioxy-substituted analogs. Pharmacol. Biochem. Behav., 58, 1109-1116.

17) Shulgin, A. (1991) PIHKAL: A Chemical Love Story, Transform Press, Berkeley, CA, U.S.A.

18) Shima, N., Katagi, M., Kamata, H., Zaitsu, K., Kamata, T., Miki, A., Tsuchihashi, H., Sakuma, T. and Nemoto, N. (2008) Conjugates of $p$-hydroxymethamphetamine and 4-hydroxy-3-methoxymethamphetamine in the blood obtained from methamphetamine and 3,4-methylenedioxymethamphetamine users: analysis by LC-MS-MS. Forensic Toxicology, 26, 58-65.

19) Miki, A., Katagi, M., Zaitsu, K., Nishioka, H. and Tsuchihashi, H. (2008) Development of a twostep injector for GC-MS with on-column derivatization, and its application to the determination of amphetamine-type stimulants (ATS) in biological specimens. J. Chromatogr. B Analyt. Tech. Biomed. Life Sci., 865, 25-32.

20) Kamata, H. T., Shima, N., Zaitsu, K., Kamata, T., Nishikawa, M., Katagi, M., Miki, A. and Tsuchihashi, H. (2007) Simultaneous analysis of new designer drug, methylone, and its metabolites in urine by gas chromatography-mass spectrometry and liquid chromatography-electrospray ionization mass spectrometry. Japanese Journal of forensic Science and Technology, 12, 97-106.

21) Miki, A., Katagi, M. and Tsuchihashi, H. (2003) Recent improvements in forensic hair analysis for illicit drugs. J. Health Sci., 49, 325-332.

22) Chèze, M., Deveaux, M., Martin, C., Lhermitte, M. and Pépin, G. (2007) Simultaneous analysis of six amphetamines and analogues in hair, blood and urine by LC-ESI-MS/MS. Application to the determination of MDMA after low ecstasy intake. Forensic Sci. Int., 170, 100-104.

23) Concheiro, M., Simões, S. M., Quintela, O., de Castro, A., Dias, M. J., Cruz, A. and López-Rivadulla, M. (2007) Fast LC-MS/MS method for the determination of amphetamine, methamphetamine, MDA, MDMA, MDEA, MBDB and PMA in urine. Forensic Sci. Int., 171, 44-51.

24) Klette, K. L., Jamerson, M. H., Morris-Kukoski, C. L., Kettle, A. R. and Suyder, J. J. (2005) Rapid simultaneous determination of amphetamine, methamphetamine, 3,4-methylenedioxyamphetamine, 3,4-methylenedioxymethamphetamine, and 3,4methylenedioxyethylamphetamine in urine by fast gas chromatography-mass spectrometry. J. Anal. Toxicol., 29, 669-674.
25) Kikura-Hanajiri, R., Kawamura, M., Saisho, K., Kodama, Y. and Goda, Y. (2007) The disposition into hair of new designer drugs; methylone, MBDB and methcathinone. J. Chromatogr. B Analyt. Tech. Biomed. Life Sci., 855, 121-126.

26) Shima, N., Kamata, H. T., Katagi, M. and Tsichihashi, H. (2006) Urinary excretion of the main metabolites of methamphetamine, including $p$-hydroxymethamphetamine-sulfate and $p$ hydroxymethamphetamine-glucuronide, in humans and rats. Xenobiotica, 36, 259-267.

27) Shima, N., Katagi, M., Kamata, H., Zaitsu, K., Kamata, T., Nishikawa, M., Miki, A., Tsichihashi, H., Sakuma, T. and Nemoto, N. (2008) Urinary excretion of the main metabolites of 3,4methylenedioxymethamphetamine (MDMA), including the sulfate and glucuronide of 4-hydroxy3-methoxymethamphetamine (HMMA), in humans and rats. Xenobiotica, 38, 314-324.

28) Kamata, H. T., Shima, N., Zaitsu, K., Kamata, T., Miki, A., Nishikawa, M., Katagi, M. and Tsuchihashi, H. (2006) Metabolism of the recently encountered designer drug, methylone, in humans and rats. Xenobiotica, 36, 709-723.

29) Zaitsu, K., Katagi, M., Kamata, T., Kamata, H., Shima, N., Tsuchihashi, H., Hayashi, T., Kuroki, H. and Matoba, R. (2008) Determination of a newly encountered designer drug " $p$-methoxyethylamphetamine" and its metabolites in human urine and blood. Forensic Sci. Int., 177, 77-84.

30) Zaitsu, K., Katagi, M., Kamata, H. T., Kamata, T., Shima, N., Miki, A., Tsuchihashi, H. and Mori, Y. (2009) Determination of the metabolites of new designer drugs bk-MBDB and bk-MDEA in human urine. Forensic Sci. Int., 188, 131-139.

31) Shima, N., Kamata, H. T., Kamata, T., Nishikawa, M., Katagi, M. and Tsuchihashi, H. (2007) The concentrations of glucuronide and sulfate of $p$-hydroxymethamphetamine in methamphetmaine users' urine, and optimization of their hydrolysis conditions. Japanese Journal of Forensic Science and Techology, 12, 73-82.

32) Pirnay, S. O., Abraham, T. T., Lowe, R. H. and Huestis, M. A. (2006) Selection and optimization of hydrolysis conditions for the quantification of urinary metabolites of MDMA. J. Anal. Toxicol., 30, 563-569.

33) Maurer, H. H., Bickeboeller-Friedrich, J., Kraemer, T. and Peters, F. T. (2000) Toxicokinetics and analytical toxicology of amphetamine-derived designer drugs ('Ecstasy'). Toxicol. Lett., 112-113, 133-142.

34) Shima, N., Tsutsumi, H., Kamata, T., Nishikawa, M., Katagi, M., Miki, A. and Tsuchihashi, H. (2006) 
Direct determination of glucuronide and sulfate of $p$-hydroxymethamphetamine in methamphetamine users' urine. J. Chromatogr. B Analyt. Tech. Biomed. Life Sci., 830, 64-70.

35) Shima, N., Kamata, H. T., Katagi, M., Tsuchihashi, H., Sakuma, T. and Nemoto, N. (2007) Direct determination of glucuronide and sulfate of 4-hydroxy-3methoxymethamphetamine, the main metabolite of MDMA, in human urine. J. Chromatogr. B Analyt. Tech. Biomed. Life Sci., 857, 123-129.

36) Shimosato, K., Tomita, M. and Ijiri, I. (1986) Urinary examination of $p$-hydroxylated methamphetamine metabolites in man. Arch. Toxicol., 59,135-140.
37) Hayakawa, K., Miyoshi, Y., Kurimoto, H., Matsushima, Y., Takayama, N., Tanaka, S. and Miyazaki, M. (1993) Simultaneous determination of methamphetamine and its metabolites in the urine samples of abusers by high performance liquid chromatography with chemiluminescence detection. Biol. Pharm. Bull., 16, 817-821.

38) Kamata, T., Zaitsu, K., Katagi, M., Suga, K., Shibayama, T., Kumihashi, M., Miyauchi, H. and Tsuchihashi, H. (2007) Analysis of urine specimens from 2,5-dimethoxyamphetamine users. The $26^{\text {th }}$ Annual Meeting of Japanese Association of Forensic Toxicology, 46-47. 